\title{
Chronic kidney disease
}

\section{Engaging our patients to change the course of this illness}

Sophia Samuel

Well about fiveyears ago, I went to the hospital because I wasn't feeling good and they took my blood pressure and it was 200 over something... Then while they were trying to get my blood pressure down, they said something about my kidneys. And I didn't know the connection between high blood pressure and kidneys. ${ }^{1}$

Chronic kidney disease (CKD) is still an under-recognised health threat in Australia. This is surprising, as it is acknowledged to be a stronger predictor of coronary disease and all-cause mortality than diabetes. ${ }^{2}$

Since the disease is nearly asymptomatic in the early stages, detecting CKD is based on opportunistically identifying, engaging with and monitoring patients or subpopulations at risk. Given these conditions, is there a rationale for general practitioners (GPs) to consider using tools such as the electronic medical record to help them case-find patients with CKD? ${ }^{2}$

CKD is not evenly spread across the population. First, there are a number of geographical areas - 'CKD hotspots' - with a much higher prevalence than others. This variation probably reflects the burden from socioeconomic disadvantage, barriers to using health services and limited access to primary care. ${ }^{3}$

Second, certain individuals are at higher risk: persons with greater susceptibility from family history or older age - or from health inequity, such as Aboriginal and Torres Strait Islander adults aged $>30$ years; those who already have nephron loss from diabetes, autoimmune conditions or high blood pressure; and those at most risk from complications or renal failure (eg people with established heart disease). ${ }^{4}$
Many of these indicators of CKD risk are entered into existing clinical information systems by practice nurses and doctors, or automatically collected from external inputs such as pathology services. The widely accepted Chronic kidney disease (CKD) management in primary care handbook recommends a whole-ofpractice strategy that includes eHealth. ${ }^{2}$ Patients accept their GPs regularly use medical software as an aide-memoire for follow-up decisions. Is there an ethical and acceptable digital search of a shared clinical information system that may identify overlooked patients who can be offered further clinical assessment?

Once early CKD and its underlying aetiology are detected, GPs can make a strong argument to their patients that this is a disease that can be altered by intervention. Lifestyle modification, tight blood pressure control and reninangiotensin system blockade preserve glomerular function in all stages. Reducing albuminuria and beginning a statin/ ezetimibe combination in people aged $>50$ years reduces cardiovascular risk independent of low-density lipoprotein levels. ${ }^{4,5}$ Patients with CKD stages 3-5 may be unaware that having a sick-day plan for their medications and fluid status will minimise acute kidney injury. ${ }^{5}$

For patients in whom kidney disease is progressive, multimorbidity, care coordination and polypharmacy become the norm. Manski-Nankervis et al present a nuanced approach to complex prescribing decisions and offer several resources to facilitate shared decision making for patients. ${ }^{6}$ One tool, the kidney failure risk equation, is a multivariable scoring system that offers a prediction of an individual's future risk of end-stage kidney disease.

Dialysis, conservative treatment and transplantation are difficult decisions made between the nephrology team and the patient. The principles of patient-centred palliative care and initiating advance care planning can help support the patients' end-of-life period. ${ }^{7}$

Patients can be empowered through understanding CKD as not confined to a single organ system but as the antecedent and consequence of several pathophysiological processes. GPs are skilled at opening this therapeutic dialogue and, when working within an adequately resourced health system, can assist patients with diagnostic and management decisions to modify their illness trajectory.

\section{Author}

Sophia Samuel FRACGP, FARGP, Medical Editor, Australian Journal of General Practice; General Practitioner, Melbourne, Vic

\section{References}

1. Roberti J, Cummings A, Myall M, et al. Work of being an adult patient with chronic kidney disease: A systematic review of qualitative studies. BM Open 2018;8:e023507. doi: 10.1136/bmjopen2018-023507.

2. Kidney Health Australia. Chronic kidney disease (CKD) management in primary care. 4th edn. Melbourne, Vic: Kidney Health Australia, 2020.

3. Australian Institute of Health and Welfare. Geographical variation in chronic kidney disease. Cat. no: WEB 191. Canberra, ACT: AIHW, 2017.

4. Wanner C, Tonelli M. KDIGO clinical practice guideline for lipid management in CKD: Summary of recommendation statements and clinical approach to the patient. Kidney Int 2014;85(6):1303-09. doi: 10.1038/ki.2014.31.

5. Usherwood T, Lee V. Advances in chronic kidney disease pathophysiology and management. Aust J Gen Pract 2021;50(4):188-92.

6. Manski-Nankervis JA, McMorrow R, Nelson C, Jesudason S, Sluggett JK. Prescribing and deprescribing in chronic kidney disease. Aust J Gen Pract 2021;50(4):183-87.

7. So S, Brennan FP, Li KC, Brown MA. End-stage kidney disease: The last 12 months. Aust J Gen Pract 2021;50(4):193-98.

correspondence ajgp@racgp.org.au 